\title{
B-Type Natriuretic Peptide Levels in Preterm Neonates With Bronchopulmonary Dysplasia: A Marker of Severity?
}

\author{
Vaneet Kumar Kalra, MD, ${ }^{1 *}$ Sanjeev Aggarwal, $\mathrm{MD}^{2}{ }^{2}$ Prem Arora, $\mathrm{MD},{ }^{1}$ and Girija Natarajan, $\mathrm{MD}^{1}$ \\ Summary. Rationale for the Study: B type natriuretic peptide (BNP) is a hormone released in \\ response to stretching of the ventricular wall. The role of BNP as a biomarker of bronchopulmonary \\ dysplasia (BPD) has not been clarified. Objective: To determine if plasma BNP concentrations \\ correlate with the severity of BPD. Methods: This prospective observational case control study \\ included 60 preterm infants ( $\leq 32$ weeks); 27 infants had no/mild BPD, 19 had moderate and 14 \\ had severe BPD. BNP levels were measured at $36 \pm 2$ weeks PMA or within a week of discharge \\ home. Groups were compared using Mann-Whitney's U-test, Kruskal-Wallis, and bivariate \\ regression. Results: Median (IQR) plasma levels of BNP in infants with moderate/severe BPD \\ infants $(n=33)$ were higher as compared to those with no/mild BPD $(n=27) ; 27.1(12.1-43.5) \mathrm{pg} /$ \\ $\mathrm{ml}$ versus 9.3 (6-18.5) $\mathrm{pg} / \mathrm{ml} ; P<0.05$ (Mann Whitney U). Median (IQR) BNP levels in infants with \\ severe BPD $(n=14), 43.5(28.4-189) \mathrm{pg} / \mathrm{ml}$ differed significantly from levels in those with \\ moderate $(n=19), 22.8(10.3-27.7) \mathrm{pg} / \mathrm{ml}$; mild $(n=16), 11.5(6.6-44.5 \mathrm{pg} / \mathrm{ml})$; or no $(n=11), 8.1$ \\ $(5-12.6 \mathrm{pg} / \mathrm{ml})$ BPD $(P<0.001 \mathrm{Kruskal}-W a l l i s)$. Based on receiver operating characteristic \\ curves, BNP $>24.4 \mathrm{pg} / \mathrm{ml}$ at $36 \pm 2$ weeks PMA or discharge home was $85.7 \%$ sensitive and \\ $76.1 \%$ specific for severe BPD. Conclusions: An elevation in plasma BNP was significantly \\ associated with severe BPD. We speculate that plasma BNP measurement in infants with BPD \\ may aid in risk-stratification and further targeted therapies. Pediatr Pulmonol. 2014; 49:1106- \\ 1111. ๑ 2013 Wiley Periodicals, Inc.
}

Key words: chronic lung disease; preterm neonates; B-type natriuretic peptide.

Funding source: Ingrid and Ashok Sarnaik Endowment fund

\section{WHAT IS KNOWN ON THIS SUBJECT}

Bronchopulmonary dysplasia (BPD) is an important cause of morbidity and mortality among premature neonates. BPD may be associated with cardiovascular sequelae. Conventional echocardiogram may not be sensitive in detecting increased pulmonary vascular pressures associated with BPD.

\section{WHAT THIS STUDY ADDS}

Infants with BPD have higher levels of B type natriurteic peptide as compared to controls. An elevation in plasma B type natriurteic peptide was significantly associated with severe BPD.

\section{BACKGROUND}

BPD is a chronic lung disease that occurs predominantly in preterm infants and, historically, is considered secondary to underdeveloped lungs and mechanical ventilation. ${ }^{1}$ Despite improvements in clinical care, the incidence of BPD has remained unchanged and a "new BPD" has evolved. ${ }^{2}$ The new BPD is characterized by interference in vascular and alveolar development and dysregulation of pulmonary vascular development with fewer pulmonary vessels and structural remodeling. ${ }^{3}$ In addition, pulmonary vessels may have increased responsiveness to oxygen $\left(\mathrm{O}_{2}\right)$, with mild hypoxia causing a marked elevation of pulmonary artery pressure. ${ }^{4}$ Therefore, infants with BPD are at risk of developing pulmonary hypertension (PHT). ${ }^{5}$ Besides pulmonary

${ }^{1}$ Division of Neonatology, The Carman and Ann Adams Department of Pediatrics, Wayne State University School of Medicine, Children's Hospital of Michigan and Hutzel Women's Hospital, Detroit, Michigan.

${ }^{2}$ Division of Pediatric Cardiology, The Carman and Ann Adams Department of Pediatrics, Wayne State University School of Medicine, Children's Hospital of Michigan and Hutzel Women's Hospital, Detroit, Michigan.

Conflict of interest: None.

${ }^{*}$ Correspondence to: Vaneet Kumar Kalra, MD, 3901 Beaubien Blvd, Ste 4C19, Detroit, MI 48201. E-mail: kalravaneet@yahoo.com

Received 30 April 2013; Accepted 23 August 2013.

DOI 10.1002/ppul.22942

Published online 8 November 2013 in Wiley Online Library

(wileyonlinelibrary.com). 
vascular changes, BPD has been shown to cause diastolic dysfunction and impaired left ventricle myocardial performance index. ${ }^{6}$

The cardiac effects of BPD are not routinely evaluated. When undertaken, echocardiography (ECHO) is used as a primary screening modality. It is limited, however, by its availability, expense and lack of sensitivity in diagnosing PHT. $^{7}$ Cardiac catheterization, considered as the gold standard for diagnosis and assessment of severity of PHT, is an invasive procedure and is only available in few tertiary centers.

B-type natriuretic peptide (BNP) is a hormone secreted by ventricular myocytes in response to stretch, that is, volume or pressure overload. It is secreted as an inactive precursor pro-BNP which is then cleaved into BNP and $\mathrm{N}$ terminal pro BNP (NT- pro BNP). BNP plays an important role in regulating systemic blood pressure and natriuresis. ${ }^{8}$ It is routinely used in the diagnosis and prognosis of congestive heart failure in adults. ${ }^{9}$ High BNP and NT- pro BNP concentrations have been previously shown in infants and children with heart disease and neonates with patent ductus arteriosus (PDA), persistent PHT, and as a guide for prophylactic therapy of PDA with indomethacin. ${ }^{10-13}$ While its use has drifted into clinical practice, there are currently scant data on BNP concentrations in preterm infants with BPD.

The purpose of this study was to evaluate the utility of BNP as a biomarker of BPD. We sought to compare plasma BNP concentrations at $36 \pm 2$ weeks postmenstrual age (PMA) or discharge home, in premature infants ( $\leq 32$ weeks gestation at birth) with and without BPD. Infants were further categorized as no, mild, moderate, and severe, according to the NIH severitybased classification and plasma BNP levels were compared among these categories. ${ }^{14}$ According to the classification, preterm infants $(\leq 32$ weeks gestation at birth) requiring supplemental oxygen at 28 days of age are diagnosed as mild BPD if they are on room air at 36 weeks PMA or discharge home, as moderate BPD if they require $<30 \%$ supplemental oxygen and as severe BPD if they require either $\geq 30 \%$ oxygen or positive pressure ventilation, including continuous positive airway pressure at 36 weeks PMA or discharge home. ${ }^{14}$ We hypothesized that plasma BNP concentrations at $36 \pm 2$ weeks PMA or discharge home would be significantly elevated in preterm neonates with BPD, compared to those without $\mathrm{BPD}$ and with increasing severity of BPD.

\section{METHODS}

\section{Patient Population}

This was a prospective observational case-control study conducted in the Neonatal Intensive Care Unit (NICU) and special care nursery at Hutzel Women's Hospital (HWH) and the NICU at Children's Hospital of Michigan (CHM).
All infants, either inborn or transferred from an outside hospital, were considered eligible for enrolment, provided their gestational age was $\leq 32$ weeks by the best obstetric estimate. Infants with congenital heart disease (except patent foramen ovale), multiple congenital anomalies, hemodynamic instability requiring pressors, active bloodstream or respiratory infections, renal insufficiency or sudden clinical deterioration were excluded from the study. The study was approved by the Institutional Review Board (IRB) at Wayne State University.

Medical records were reviewed to determine eligibility and seek parental consent. Once determined to be eligible, parents were approached for a written consent. Study procedures included blood draw of $0.25 \mathrm{ml}$ for measurement of BNP concentration at $36 \pm 2$ weeks PMA or discharge home.

\section{Measurement of Plasma B-Type Natriuretic Peptide}

BNP was measured using the Food and Drug Administration-approved Biosite Triage BNP kits (Biosite Diagnostics Inc., Alere, San Diego, CA). Its interassay imprecision is $9.9-12.2 \%$. The assay, which utilizes a disposable device, was performed by a single investigator (V.K.) after a brief training and took approximately $15 \mathrm{~min}$. Whole blood $(0.25 \mathrm{ml})$ is added to the sample port of the device. The filter in the device separates the blood cells from the plasma. The separated plasma migrates through the diagnostic lane with the help of capillary action and reacts with fluorescence-tagged murine polyclonal antibodies against the ring structure of BNP. Excess plasma washes away the unbound antibody. An internal calibration curve is used to quantify the fluorescence intensity of the BNP assay zone.

The medical records were reviewed for data abstraction. Data collected included infant demographics, birth weight and its category (appropriate, small or large-for gestational age, mode of delivery and Apgar score at 5 min. $^{15}$ Maternal data (ethnicity, use of antenatal steroids, duration of rupture of membranes, and chorioamnionits) were also collected. Clinical data including age when BNP was measured, days of $\mathrm{O}_{2}$ or ventilation requirement, morbidities such as necrotizing enterocolitis (NEC), intraventricular hemorrhage (IVH), severe IVH (grade 3 or 4), PDA requiring treatment, effective $\mathrm{O}_{2}$ delivery, and medications for PHT were reviewed. ${ }^{16,17} \mathrm{In}$ ventilated patients, peak respiratory severity scores (RSS) were calculated using standard formula as the product of mean airway pressure and fraction of inspired $\mathrm{O}_{2}$ on the day of BNP sampling and 2 and 7 days earlier. ${ }^{18}$ BPD severity was graded as no, mild, moderate and severe according to the severity-based classification of National Institute of Health (NIH). ${ }^{14}$ 
Cardiac imaging was not done specifically for this study. Reports of echocardiograms (ECHOs) performed for clinical care were reviewed to rule out congenital heart disease (except PFO) and myocardial dysfunction. Cardiac catheterization, done at any time, was reviewed for presence of PHT.

\section{Statistical Analysis}

Reported mean \pm SD plasma BNP concentrations at $1-$ 6 months of life are $21 \pm 10 \mathrm{pg} / \mathrm{ml} .{ }^{19}$ We expected a $25 \%$ difference in the BNP values between patients with BPD and controls, based on a 50\% higher BNP level in patients with stable chronic obstructive pulmonary disease without any evidence of PHT, compared to controls. ${ }^{20}$ For an alpha (for the two-tailed test) of 0.05 and beta of 0.2 , a sample size of 27 in each group was calculated to give the study a power of $80 \%$ and yield a statistically significant result.

Data analysis was performed with SPSS 19 (SPSS, Inc., Chicago, IL). Descriptive data included mean (SD), median (interquartile range) and number (percent) as appropriate. Chi square test was used to compare descriptive variables between groups. The Kolmogorov-Smirnov test of normality showed a non-normal distribution of continuous variables and therefore, nonparametric tests (Mann-Whitney $U$, Spearman correlation, Kruskal-Wallis) were used for further comparisons. BNP was evaluated as a marker of BPD using area under receiver operating characteristic (ROC) curves. Regression analysis was used to examine the association between BPD and gestational age, C-section and PDA (variables found to be significantly different in bivariate comparisons) and BNP. A $P$-value of $<0.05$ was taken as statistically significant.

\section{RESULTS}

A total of 60 preterm infants were studied; 40 (66.7\%) infants were enrolled from the Level III inborn unit at HWH and 20 (33.4\%) were enrolled from the regional referral Level IV NICU at CHM. Figure 1 is a description of patient accrual. The 20 infants enrolled at CHM were derived from 49 infants who met study eligibility criteria and were at CHM NICU at $36 \pm 2$ weeks PMA or discharge home. These infants were transferred from other Level III NICUs for the management of complex medical or surgical concerns such as airway evaluation for failure to extubate and placement of ventriculoperitoneal shunt.

The cohort was predominantly male (58.3\%), appropriate for gestational age (81.7\%), African-American $(68.3 \%)$, and delivered via $\mathrm{C}$ section $(65 \%)$. The median (IQR) gestational age and birth weight were 26 (24-28) weeks and $885(700-1,125) \mathrm{g}$, respectively. Antenatal steroids were administered in $53(88.3 \%)$ infants, with a

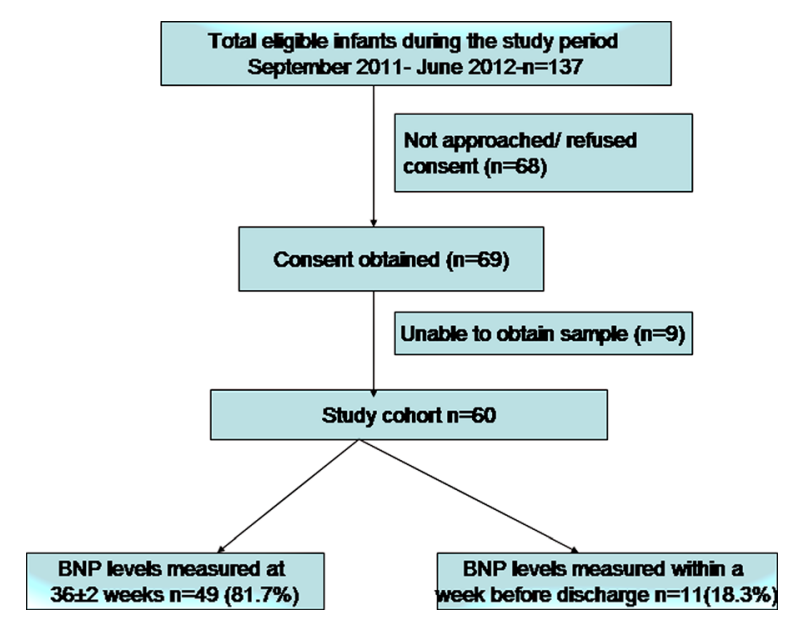

Fig. 1. Flow diagram of eligible patients.

complete course in $40(67 \%)$ cases. Ten $(16.7 \%)$ mothers had signs of clinical chorioamnionitis and $25(41.7 \%)$ had histologic chorioamnionitis. Apgar scores $\leq 5$ at $5 \mathrm{~min}$ of life were noted in 12 (20\%) infants. Median (IQR) surfactant doses and weight at the time of BNP measurement were 1 (1-2.75) and $1,922(1,770$ $2,221) \mathrm{g}$, respectively. Of our samples, $24 / 60$ were prior to 36 weeks PMA at the time of discharge and $15 / 60$ were drawn subsequent to 36 weeks. The median (IQR) PMA at sampling was 36 (35-37) weeks. An ECHO was performed at some point during hospitalization in 45 (75\%) infants; only 14 (23\%) underwent an ECHO within 14 days of BNP measurement. Of the 33 infants with BPD, 12 (36\%) had an ECHO within 14 days, and 32 (97\%) had an ECHO at some point during their NICU stay. A PDA was documented to be absent on the latest ECHO or was surgically ligated in $30(91 \%)$ of the infants with BPD. The latest echocardiogram showed a tiny PDA in two infants. Two infants, who had findings suggestive of elevated pulmonary pressures on cardiac catheterization had BNP levels of 324 and $400 \mathrm{pg} / \mathrm{ml}$.

Of the study cohort, $33(55 \%)$ infants required supplemental $\mathrm{O}_{2}$ at 36 weeks PMA or discharge home and were characterized as having BPD, whereas 27 (45\%) infants were in the no BPD group. Table 1 is a description of the respiratory support $-\mathrm{O}_{2}$ delivered and RSS in infants with and without BPD at the time of BNP measurement and 2 and 7 days prior to BNP measurement. The lack of significant differences over time suggest stable respiratory support at the time of BNP measurement. Two infants without BPD were on $\mathrm{O}_{2}$ at the time of BNP sampling (PMA 33 4/7 weeks and 36 0/7 weeks); both were discharged home without $\mathrm{O}_{2}$ within a week of sampling and before completion of 36 weeks PMA. At BNP measurement, 13 (21.7\%) infants were receiving antimicrobial therapy but none had positive cultures from any site for more than a week. All infants 
TABLE 1-Respiratory Support in Infants With and Without BPD at the Time of BNP Measurement, 2 and 7 Days Prior to BNP Measurement

\begin{tabular}{|c|c|c|c|}
\hline Median (IQR) & BNP measurement day & 2 days prior & 7 days prior \\
\hline \multicolumn{4}{|c|}{$\mathrm{O} 2$ delivery (lpm) } \\
\hline BPD & $0.26(0.24-0.34), \mathrm{n}=21$ & $0.26(0.24-0.29), \mathrm{n}=20$ & $0.26(0.25-0.30), \mathrm{n}=19$ \\
\hline No BPD & $0.25, \mathrm{n}=2$ & $0.23(0.215-0.27), \mathrm{n}=2$ & $0.24(0.22-0.27), \mathrm{n}=3$ \\
\hline \multicolumn{4}{|l|}{ RSS } \\
\hline BPD & $2.46(2.5-5.5), \mathrm{n}=12$ & $3.2(2.49-5.14), \mathrm{n}=13$ & $3.2(2.32-4.49), \mathrm{n}=12$ \\
\hline No BPD & $\mathrm{N}=0$ & $\mathrm{~N}=0$ & $3.0, \mathrm{~N}=1$ \\
\hline
\end{tabular}

$\mathrm{O}_{2}$, oxygen; BPD, bronchopulmonary dysplasia; BNP, B-type natriuretic peptide; RSS, respiratory severity score.

had good urine output with a median (IQR) serum creatinine of $0.3(0.2-0.4) \mathrm{mg} / \mathrm{dl}$. Table 2 is a comparison of the clinical characteristics between the groups with and without $\mathrm{BPD}$, defined as $\mathrm{O}_{2}$ supplementation at 36 weeks PMA. Median BNP concentrations were significantly elevated in the BPD group (27.1 IQR: $12.1-43.5$ vs. 9.3 IQR: $6-18.5 \mathrm{pg} / \mathrm{ml}, P=0.003$ by Mann-Whitney $U$ ), compared to the no BPD group. Infants with BPD were significantly more immature, more often African-American, had lower birth weights and had longer $\mathrm{O}_{2}$ and ventilation need and higher rates of PDA requiring treatment, compared to those without BPD. Five (8.3\%) infants with BPD required a tracheostomy and home ventilation and two $(3.3 \%)$ infants died. Binary regression analysis was used to examine the association between $\mathrm{BNP}$ concentration and BPD, with gestational age, $\mathrm{C}$ section, and PDA (the variables found to be significantly different in bivariate comparisons) as covariates. Gestational age, although not BNP, tended to be associated with BPD (OR 0.71; 95\% CI 0.49-1.01; $P=0.056$ ).

Based on the NIH classification of severity of BPD, 14/ 60 infants had severe, 19 had moderate, and 16 infants had mild BPD. Eleven patients were not on any supplemental oxygen at 28 days of life and were characterized as no BPD. Median (IQR) BNP concentrations in the group with severe BPD (43.5 IQR: 28.4-189 pg/ml) was significantly elevated, compared to levels in groups with moderate BPD (22.8 IQR: $10.3-27.7 \mathrm{pg} / \mathrm{ml}$ ), mild BPD (11.15 IQR: $6.6-44.5 \mathrm{pg} / \mathrm{ml})$, or no BPD (8.1 IQR:5-12.6 pg/ml $) \quad(P<0.001$ Kruskal-Wallis $)$. There were no significant differences in BNP values between those with moderate, mild, and no BPD. Figure 1 is a box plot showing the median (IQR) BNP values in these four categories.

We then performed regression analysis to examine the association between severe BPD and gestational age, $\mathrm{C}$ section, PDA, and BNP concentrations. BNP concentration (OR 1.05; 95\% C.I 1.005-1.098; $P=0.028$ ) was independently associated with severe BPD, while gestational age $(P=0.073)$ and PDA $(P=0.853)$ were not. Plasma BNP at $36 \pm 2$ weeks PMA at a cut-off value of $24.4 \mathrm{pg} / \mathrm{ml}$ had an area under the ROC curve of 0.8 (95\% CI $0.64-0.97)$, sensitivity of $85.7 \%$ and specificity of $76.1 \%$ (Fig. 2).

TABLE 2-Clinical Characteristics of the Groups With and Without BPD

\begin{tabular}{lccc}
\hline Variable n (\%) or median (IQR) & No BPD $(\mathrm{n}=27)$ & BPD $(\mathrm{n}=33)$ & $P$-value \\
\hline Males & $13(48 \%)$ & $22(66.67 \%)$ & 0.19 \\
Gestational age (weeks) & $28.1(26.9-30.6)$ & $25.3(24-26.7)$ & $<0.001$ \\
Birth weight (g) & $1,110(940-1,250)$ & $720(615-875)$ & $<0.001$ \\
Small for Gestational Age & $2(7.4 \%)$ & $5(15.2 \%)$ & 0.65 \\
African-Americans & $14(51.9 \%)$ & $27(81.8 \%)$ & 0.09 \\
Any antenatal steroids & $22(81.5 \%)$ & $31(93.9 \%)$ & 0.23 \\
Maternal Histologic Chorioamnionits & $11(40.8 \%)$ & $14(42.4 \%)$ & 0.71 \\
PPROM $>18$ hr & $7(25.9 \%)$ & $9(27.3 \%)$ & 1.00 \\
Apgar score at 5 minutes $\leq 5$ & $3(11.1 \%)$ & $9(27.3 \%)$ & 0.20 \\
Inborn & $18(66.7 \%)$ & $22(66.7 \%)$ & 1.00 \\
C-section & $22(81.5 \%)$ & $17(51.5 \%)$ & 0.03 \\
Age at BNP (day of life) & $53(29-61)$ & $92(64.5-97.5)$ & $<0.001$ \\
Oxygen days & $30(8-60)$ & $60.5(15.3-78)$ & $<0.001$ \\
Ventilation days & $2(1-10)$ & $30(12-75.5)$ & $14(42.4 \%)$ \\
Age extubated (days) & $2(0-8)$ & $8(24.2 \%)$ & 0.001 \\
Any IVH & $11(40.8 \%)$ & $12(36.4 \%)$ & 1.001 \\
Severe IVH (Grade 3 or 4) & $4(14.8 \%)$ & $19(57.6 \%)$ & 0.09 \\
NEC & $6(22.2 \%)$ & $9.3(6-18.5)$ & 0.27 \\
PDA & $6(22.2 \%)$ & 0.008 \\
BNP level (pg/ml) & $27.1(12.1-75.5)$ & 0.003 \\
\hline
\end{tabular}



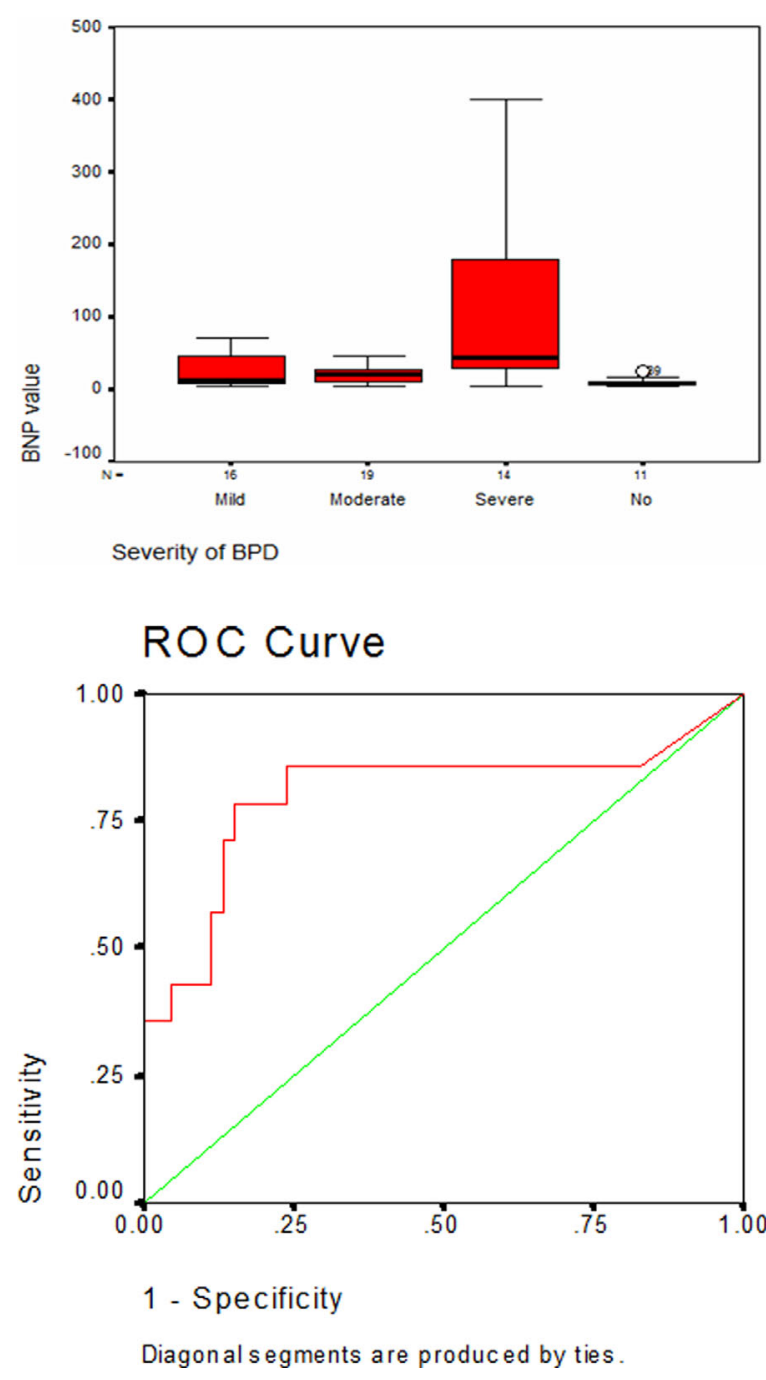

Fig. 2. BNP levels in four different groups of BPD severity.

\section{DISCUSSION}

In this study, we found that BNP at $36 \pm 2$ weeks PMA or discharge home was significantly higher in preterm infants with BPD, compared to those without BPD and in those with severe BPD, compared to others. BNP retained a significant independent association with severe BPD, after adjustment for gestational age and clinical differences.

Our results correlate with the findings of Joseph et al., ${ }^{21}$ who, in an observational pilot study of 11 infants, reported higher NT-pro-BNP levels in preterm infants with BPD and a correlation between levels and clinical severity of respiratory disease. Cuna et al. ${ }^{22}$ in their retrospective study of 25 preterm infants also reported higher BNP levels in BPD infants with ECHO evidence of PHT. Our findings, however, are contradictory to the findings of Akcan et al. who, in a study of 28 subjects, did not find any significant difference in NT-proBNP levels among patients with a history of BPD compared to controls and among infants with varying severity of BPD. ${ }^{23}$ This difference in results is probably related to the time of measurement, which in Akcan's study was at a mean postnatal age of 9.8 months when most of the clinical findings of BPD become less severe.

In contrast to previous studies, our study measured plasma BNP at a consistent time of $36 \pm 2$ weeks PMA. This later time was selected to examine the utility of BNP as a marker of cardiac effects of BPD, which would have developed over time and second, to allow the effects of respiratory distress syndrome severity to wane. It also obviated the initial postnatal changes in BNP concentrations. ${ }^{19}$ The interaction between gestational age, BNP concentrations and BPD is complex. As expected, gestational age of the group with BPD differed significantly from the controls. To account for this, a consistent PMA was selected for sampling. Moreover previous studies have reported comparable BNP levels in preterm and term infants after 28 days of life. ${ }^{19,24}$

The mechanism of elevation in BNP in BPD is not entirely clear. BNP has been previously shown to be a counter-regulatory hormone released in response to sustained pulmonary hypoxia. ${ }^{25}$ It induces pulmonary relaxation and plays a role in modulating the pulmonary hypertensive response to chronic hypoxia. ${ }^{26}$ In addition, BNP is considered a biomarker of disease severity and prognosis in adult patients with PHT. ${ }^{27}$ Elevated BNP levels are seen in adult patients with stable chronic obstructive pulmonary disease without evidence of PHT. ${ }^{20}$ In contrast, among infants with acute respiratory distress, plasma NT-pro BNP levels were significantly increased in those with heart failure, compared to those with lung disease. ${ }^{28}$ Elevation in BNP has been demonstrated in adults with diastolic dysfunction. ${ }^{29}$ Therefore, elevated BNP in BPD could be related to increased pulmonary vascular pressures, chronic pulmonary disease itself, diastolic dysfunction or impaired global left ventricular function. 6,12

We recognize the limitations of our study which include small sample size, range of gestational ages, lack of ECHO at a defined time point in all infants and some baseline differences between groups. Nonetheless, we undertook a systematic evaluation of the utility of BNP in BPD, defined by the standardized NIH-severity based classification and found novel associations with severe BPD. We meticulously excluded factors known to affect plasma BNP such as active infections, renal insufficiency and worsening respiratory condition.

PHT is an increasingly recognized accompaniment of BPD and is associated with increased mortality and morbidity. ${ }^{30}$ Collaco et al. $^{30}$ have underscored the "extreme phenotypic variability that exists among preterm infants of similar gestational ages, making it 
difficult to predict which infants are at increased risk for developing PHT." Many experts recognize that sensitive and specific, rapidly measured bedside biomarkers are needed to improve the diagnosis and management of cardiac sequelae of BPD in preterm children. Such biomarkers may potentially aid in the prognostication, risk-stratification and targeted therapy in those at highest risk. We believe the current study is a first step in this direction. Further studies of serial BNP measurement for prediction or early detection of severe BPD are warranted.

\section{REFERENCES}

1. Northway WH, Jr., Rosan RC, Porter DY. Pulmonary disease following respirator therapy of hyaline-membrane disease. Bronchopulmonary dysplasia. N Engl J Med 1967;276:357-368.

2. Jobe AH. What is BPD in 2012 and what will BPD become? Early Hum Dev 2012;88:S27-S28.

3. Jobe AJ. The new BPD: an arrest of lung development. Pediatr Res Dec 1999;46:641-643.

4. Mourani PM, Ivy DD, Gao D, Abman SH. Pulmonary vascular effects of inhaled nitric oxide and oxygen tension in bronchopulmonary dysplasia. Am J Respir Crit Care Med 2004;170:1006-1013.

5. Khemani E, McElhinney DB, Rhein L, Andrade O, Lacro RV, Thomas KC, Mullen MP. Pulmonary artery hypertension in formerly premature infants with bronchopulmonary dysplasia: clinical features and outcomes in the surfactant era. Pediatrics 2007;120:1260-1269.

6. Yates AR, Welty SE, Gest AL, Cua CL. Myocardial tissue Doppler changes in patients with bronchopulmonary dysplasia. J Pediatr 2008;152:766-770, 770 e761.

7. Mourani PM, Sontag MK, Younoszai A, Ivy DD, Abman SH. Clinical utility of echocardiography for the diagnosis and management of pulmonary vascular disease in young children with chronic lung disease. Pediatrics 2008;121:317-325.

8. Levin ER, Gardner DG, Samson WK. Natriuretic peptides. N Engl J Med 1998;339:321-328.

9. Gotze JP, Kastrup J. Plasma pro-brain natriuretic peptides are strong biochemical markers in clinical cardiology. Scand J Clin Lab Invest Suppl 2001;234:47-51.

10. Law YM, Hoyer AW, Reller MD, Silberbach M. Accuracy of plasma B-type natriuretic peptide to diagnose significant cardiovascular disease in children: the Better Not Pout Children! Study. J Am Coll Cardiol 2009;54:1467-1475.

11. Kalra VK, DeBari VA, Zauk A, Kataria P, Myridakis D, Kiblawi F. Point-of-care testing for B-type natriuretic peptide in premature neonates with patent ductus arteriosus. Ann Clin Lab Sci Spring 2011;41:131-137.

12. Vijlbrief DC, Benders MJ, Kemperman H, van Bel F, de Vries WB. B-type natriuretic peptide and rebound during treatment for persistent pulmonary hypertension. J Pediatr 2012;160:111-115, e111.

13. Nuntnarumit P, Chongkongkiat P, Khositseth A. N-terminal-probrain natriuretic peptide: a guide for early targeted indomethacin therapy for 1patent ductus arteriosus in preterm Infants. Acta Paediatr 100:1217-1221.
14. Jobe AH, Bancalari E. Bronchopulmonary dysplasia. Am J Respir Crit Care Med 2001;163:1723-1729.

15. Olsen IE, Groveman SA, Lawson ML, Clark RH, Zemel BS. New intrauterine growth curves based on United States data. Pediatrics 2010;125:e214-e224.

16. Papile LA, Burstein J, Burstein R, Koffler H. Incidence and evolution of subependymal and intraventricular hemorrhage: a study of infants with birth weights less than 1,500 gm. J Pediatr 1978;92:529-534.

17. Walsh M, Engle W, Laptook A, Kazzi SN, Buchter S, Rasmussen M, Yao Q. Oxygen delivery through nasal cannulae to preterm infants: can practice be improved? Pediatrics 2005;116: 857-861.

18. Ballard RA, Truog WE, Cnaan A, Martin RJ, Ballard PL, Merrill JD, Walsh MC, Durand DJ, Mayock DE, Eichenwald EC. Inhaled nitric oxide in preterm infants undergoing mechanical ventilation. N Engl J Med 2006;355:343-353.

19. Cantinotti M, Storti S, Parri MS, Murzi M, Clerico A. Reference values for plasma B-type natriuretic peptide in the first days of life. Clin Chem 2009;55:1438-1440.

20. Inoue Y, Kawayama T, Iwanaga T, Aizawa H. High plasma brain natriuretic peptide levels in stable COPD without pulmonary hypertension or cor pulmonale. Intern Med 2009;48:503-512.

21. Joseph L, Nir A, Hammerman C, Goldberg S, Ben Shalom E, Picard E. N-terminal pro-B-type natriuretic peptide as a marker of bronchopulmonary dysplasia in premature infants. Am J Perinatol 2010;27:381-386.

22. Cuna A, Kandasamy J, Fineberg N, Sims B. B-type natriuretic peptide is a biomarker for pulmonary hypertension in preterm infants with bronchopulmonary dysplasia. Res Rep Neonatol 2013;3:33-36

23. Akcan AB, Kardelen F, Oygucu SE, Kocabaş A, Ozel D, Akbaş H, Oygür N. The efficacy of cardiac findings in assessing the outcome in preterms with bronchopulmonary dysplasia. Indian J Pediatr 2013. DOI 10.1007/s12098-013-0994-y

24. Mannarino S, Garofoli F, Mongini E, et al. BNP concentrations and cardiovascular adaptation in preterm and fullterm newborn infants. Early Hum Dev 2010;86:295-298.

25. Hill NS, Klinger JR, Warburton RR, Pietras L, Wrenn DS. Brain natriuretic peptide: possible role in the modulation of hypoxic pulmonary hypertension. Am J Physiol 1994;266:L308-315.

26. Klinger JR, Warburton RR, Pietras L, Hill NS. Brain natriuretic peptide inhibits hypoxic pulmonary hypertension in rats. J Appl Physiol 1998;84:1646-1652.

27. Nagaya N, Nishikimi T, Uematsu M, et al. Plasma brain natriuretic peptide as a prognostic indicator in patients with primary pulmonary hypertension. Circulation 2000;102:865-870.

28. Cohen S, Springer C, Avital A, Perles Z, Rein AJ, Argaman Z, Nir A. Amino-terminal pro-brain-type natriuretic peptide: heart or lung disease in pediatric respiratory distress? Pediatrics 2005;115:1347-1350.

29. Parekh N, Maisel AS. Utility of B-natriuretic peptide in the evaluation of left ventricular diastolic function and diastolic heart failure. Curr Opin Cardiol 2009;24:155-160.

30. Collaco JM, Romer LH, Stuart BD, et al. Frontiers in pulmonary hypertension in infants and children with bronchopulmonary dysplasia. Pediatr Pulmonol 2012;47:1042-1053. 